\title{
Effect of Time and Fatty Acid Composition in Eggs of White Leghorn Hens Supplemented with Tuna Oil
}

\author{
Jesus Eduardo Morales-Barrera ${ }^{1}$, Mariano Jesus Gonzalez-Alcorta ${ }^{2}$, Rosa Maria Castillo-Dominguez ${ }^{3}$, \\ Omar Francisco Prado-Rebolledo ${ }^{4}$ Jose Luis Vazquez ${ }^{2}$, Xochitl Hernandez-Velasco, \\ Guillermo Tellez ${ }^{6}$, Anita Menconi ${ }^{6}$, Billy Marshal Hargis ${ }^{6}$, Silvia Carrillo-Dominguez ${ }^{3 *}$ \\ ${ }^{1}$ Departamento de Producción Agrícola y Animal, Universidad Autónoma Metropolitana, México City, México; ${ }^{2}$ Universidad \\ Autónoma Chapingo, Estado de México, México; ${ }^{3}$ Depto. Nutrición Animal, Instituto Nacional de Ciencias Médicas y Nutrición \\ Salvador Zubirán, México City, México; ${ }^{4}$ Facultad de Medicina Veterinaria y Zootecnia, Universidad de Colima, Colima, México; \\ ${ }^{5}$ Facultad de Medicina Veterinaria y Zootecnia, Universidad Nacional Autónoma de México, México City, México; ${ }^{6}$ Department of \\ Poultry Science, University of Arkansas, Fayetteville, USA. \\ Email: *silvicarrillo3@hotmail.com
}

Received May $16^{\text {th }}, 2013$; revised June $16^{\text {th }}, 2013$; accepted June $25^{\text {th }}, 2013$

Copyright (C) 2013 Jesus Eduardo Morales-Barrera et al. This is an open access article distributed under the Creative Commons Attribution License, which permits unrestricted use, distribution, and reproduction in any medium, provided the original work is properly cited.

\begin{abstract}
The study evaluated the effect of time and fatty acid (FA) composition in eggs of laying hens supplemented with tuna oil (TO). Two hundred White Leghorn hens 30-week were divided into 5 treatments with 4 replicates of 10 birds each. Treatments were assigned randomly and consisted of $0 \%, 0.75 \%, 1.50 \%, 2.25 \%$ and $3 \%$ TO in commercial sorghumsoybean meal diets, in which the soybean oil was partially replaced. The experiment was conducted for 90 days. At days 28, 56 and 84 of the experiment, 10 eggs per replicate were collected for chemical analysis. Feed intake, egg production rate, egg weight, and feed conversion were not influenced by dietary treatment. FA content was significantly altered $(\mathrm{P}<0.05)$ by TO, showing a progressive increase in egg n-3 FA (especially docosahexaenoic [DHA] and eicosapentaenoic [EPA] acids) when TO was added. Levels of EPA and DHA were higher $(\mathrm{P}<0.05)$ in the egg lipids of TO fed hens than those in the control group. This was correlated $(\mathrm{P}<0.05)$ with the concentration of TO in the diet. However, no significant differences were observed either in egg weight or percent of egg lay between groups. There was a high correlation $(\mathrm{P}<0.05)$ between TO inclusion level in the diet and the incorporation of EPA $(\mathrm{r}=0.96)$ or DHA $(r=0.92)$ into the egg yolk and an overall decrease in $n-6$ FA. There was a high correlation $(P<0.05)$ between TO inclusion level and the incorporation of linoleic acid (LA, $r=-0.95)$ or arachidonic acid (AA, $r=-0.96)$ into the egg yolk. The highest incorporation $(\mathrm{P}<0.05)$ of total $\mathrm{n}-3 \mathrm{FA}$ content in eggs was obtained with $3 \% \mathrm{TO} / \mathrm{kg}$. This increase was proportional to TO inclusion levels in the diets $(r=0.95)$. The results indicate that the $n-3$ FA content in eggs can be increased by dietary supplementation with TO, and that TO can serve as a reasonable alternative feed ingredient in layer diets to produce a healthier choice of egg.
\end{abstract}

Keywords: Egg Enrichment; Laying Hens; n-3 Fatty Acids; Tuna Oil

\section{Introduction}

Recently, there has been much scientific and public interest in elucidating the role of diet in preventing morbidity and mortality resulting from some types of cancer, auto immune and cardiovascular diseases [1-4]. The World Health Organization recommends that diets should pro- vide less than $1 \%$ of total energy intake as trans fatty acids [5]. Traditionally, Western diets are de-

${ }^{*}$ Corresponding author. ficient in excessive amounts of omega-6 PUFA [4]. In many countries, the consumption of marine products is low; hence the benefit that could be derived from a diet rich in n-3 PUFA does not reach the majority of the population. Fatty acid (FA) content of the egg yolk can be modified through nutrition. Currently, linseed oil and marine products are used commercially to achieve this effect [6-11]. The aim of the present study was to evaluate the effect of length of supply and FA composition in eggs of White Leghorn hens supplemented with tuna oil. 


\section{Materials and Methods}

\subsection{Birds and Diets}

Two hundred White Leghorn hens were divided into five treatments with four replicates of ten birds each, housed in individual cages. The age of the hens at the start of the experiment was 30 weeks and an average body weight of $1858 \pm 80 \mathrm{~g}$. The treatments were assigned randomly and consisted of the incorporation of $0.75 \%, 1.5 \%, 2.25 \%$ and 3\% in substitution of identical amount of soya oil in commercial sorghum-soybean diets. The diets (Table 1) were calculated to meet the recommendations of the National Research Council Poultry [12] and the experiment lasted 90 days (three month production periods). Diets were formulated based on chemical analysis of fatty acid $n-3$ and $n-6$ profiles in the soybean oil and TO used in this study (Table 2). Feed mixtures were made fresh just prior to each month production period. Feed and water were offered for ad libitum consumption. Data from feed intake, egg production rate, egg weight, and feed conversion were calculated and egg samples were collected over a month production period.

\subsection{Analytical Methods}

At days 28,56 and 84 (1, 2, and 3 months respectively) after the study started, 10 eggs from each replicate $(40$ per group) were collected and stored at $4^{\circ} \mathrm{C}$ for whole egg (yolk and albumen) chemical analysis. Three replicate $1 \mathrm{~g}$ aliquots of each sample were processed according to the methods described by Folch et al. (1957) [13]. Methyl esters of fatty acids were obtained using boron trifluoride. Fatty acids were quantified by gas chromatography using a DB-23 column (JW 122-2332 of $30 \mathrm{~m} \times$ $0.25 \mathrm{~mm}$ internal diameter) on a Varian $3400 \mathrm{CX}$ gasliquid chromatograph, equipped with an auto sampler and a flame ionization detector (Varian Associates, Inc., Sugar Land, TX). Nitrogen was the carrier gas at a flow rate of $30 \mathrm{~mL} / \mathrm{min}$. Temperatures were: column, $230^{\circ} \mathrm{C}$; injector, $150^{\circ} \mathrm{C}$; detector, $300^{\circ} \mathrm{C}$. Myristic acid (Sigma Co., St. Louis, MO) was used as an internal standard for FA. Retention times were compared with FA methyl ester standards [14].

\subsection{Statistical Analysis}

Data from productive performance and PUFA content in the egg were evaluated by analysis of variance using the General Linear Models (GLM) procedure. Significant differences $(\mathrm{P}<0.05)$ were further separated using Duncan's multiple range test and commercial statistical analysis software, with length of supply and treatment as main effects. Regression analysis between the inclusion of TO and n-3 FA and n-6 FA concentration in the egg content was also evaluated using commercial statistical analysis software [15].

\section{Results and Discussion}

\subsection{Production Performance}

The use of TO did not affect productive performance of the laying hens (Table 3), in agreement with Baucells et al., (2000) [8] who incorporated different PUFA into hens' diets without finding any effect.

\subsection{Fatty Acid Composition}

Fatty acid composition of egg yolk is readily altered by dietary manipulation [8]. FA content was altered by TO inclusion with a progressive increase in egg n-3 PUFA, especially docosahexaenoic acid (DHA, C22:6) and eicosapentaenoic acid (EPA, C20:5) whit increasing levels. The increase was associated with a progressive decrease in alpha-linolenic acid (ALA, C18:3), a precursor of the more biologically active forms of n-3, EPA and DHA. Levels of EPA were higher $(\mathrm{P}<0.05)$ in the egg lipids of hens fed TO than those in the control group. Likewise, eggs from hens fed TO had higher DHA content than the control eggs. There was a high correlation between the TO inclusion level in the diets and the incorporation of EPA $(r=0.96)$ or DHA $(r=0.92)$ into the egg yolk $($ Table 4).

In the present study, a progressive decrease in linoleic acid (LA, C18:2) and arachidonic acid (AA, C20:4) was observed when TO was added (Table 5). There was a high correlation $(\mathrm{P}<0.05)$ between the TO inclusion level in the diets and the incorporation of LA $(r=-0.95)$ or AA $(r=-0.96)$ into the egg yolk. The highest incurporation of total n-3 FA content in eggs was obtained with 3\% TO (Table 6). This increase was proportional to $\mathrm{TO}$ inclusion levels in the diets $(\mathrm{r}=0.95)$.

Among the fatty acids, n-3 PUFA which has been described to have the most effective immunomodulatory activities, and among the n-3 PUFA, those from fish oilEPA and DHA are more biologically potent than ALA $[2,16]$. Current Western diets typically contains excessive amounts of n-6 PUFA and high omega-6/omega-3 ratio which have been associated with several pathological conditions such as cardiovascular disease, cancer, and inflammatory and autoimmune diseases, whereas increased levels of omega-3 PUFA have been reported to reduce those conditions [17-20].

\section{Conclusion}

Our results indicate that the n-3 PUFA content in eggs can be increased by dietary supplementation with TO and that TO serves as an energy and n-3 fatty acids alternative feed ingredient in layer hen diets to produce a healthier choice of egg. Addition of TO to laying hen 
Table 1. Analysis and percentage composition of the experimental diets with different levels of tuna oil (\%).

\begin{tabular}{|c|c|c|c|c|c|}
\hline \multirow{2}{*}{ Item } & \multicolumn{5}{|c|}{ Tuna oil (\%) } \\
\hline & 0 & 0.75 & 1.50 & 2.25 & 3 \\
\hline \multicolumn{6}{|l|}{ Ingredient } \\
\hline Sorghum & 62.54 & 62.54 & 62.54 & 62.54 & 62.54 \\
\hline Soybean meal & 23.85 & 23.85 & 23.85 & 23.85 & 23.85 \\
\hline Soybean oil & 3.00 & 2.25 & 1.50 & 0.75 & 0.00 \\
\hline Tuna oil & 0.00 & 0.75 & 1.50 & 2.25 & 3.00 \\
\hline Calcium carbonate & 8.33 & 8.33 & 8.33 & 8.33 & 8.33 \\
\hline Calcium phosphate & 1.28 & 1.28 & 1.28 & 1.28 & 1.28 \\
\hline Salt & 0.40 & 0.40 & 0.40 & 0.40 & 0.40 \\
\hline Mineral Mix ${ }^{1}$ & 0.10 & 0.10 & 0.10 & 0.10 & 0.10 \\
\hline Vitamin $\mathrm{Mix}^{2}$ & 0.25 & 0.25 & 0.25 & 0.25 & 0.25 \\
\hline Coline chloride $60 \%$ & 0.05 & 0.05 & 0.05 & 0.05 & 0.05 \\
\hline DL-methionine & 0.05 & 0.05 & 0.05 & 0.05 & 0.05 \\
\hline Pigment $^{3}$ & 0.15 & 0.15 & 0.15 & 0.15 & 0.15 \\
\hline Total & 100.0 & 100.0 & 100.0 & 100.0 & 100.0 \\
\hline \multicolumn{6}{|l|}{ Calculated analysis } \\
\hline ME (Mcal) & 2857 & 2850 & 2842 & 2835 & 2827 \\
\hline $\mathrm{CP}, \%$ & 16.00 & 16.00 & 16.00 & 16.00 & 16.00 \\
\hline Lysine, $\%$ & 0.77 & 0.77 & 0.77 & 0.77 & 0.77 \\
\hline Methionine, $\%$ & 0.30 & 0.30 & 0.30 & 0.30 & 0.30 \\
\hline Methionine + cysteine, $\%$ & 0.59 & 0.59 & 0.59 & 0.59 & 0.59 \\
\hline Calcium, \% & 3.50 & 3.50 & 3.50 & 3.50 & 3.50 \\
\hline Available phosphorus, \% & 0.35 & 0.35 & 0.35 & 0.35 & 0.35 \\
\hline
\end{tabular}

${ }^{1}$ Vitamin premix supplied the following per kilogram of complete feed: vitamin A, 12,000 IU; vitamin D3, 2500 IU; vitamin E, 30 IU; vitamin K3, 2 mg; thiamine, $2.25 \mathrm{mg}$; riboflavin, $7.5 \mathrm{mg}$; pyridoxine, $3.5 \mathrm{mg}$; cobalamine, $0.02 \mathrm{mg}$; niacin, $45 \mathrm{mg}$; D-pantothenic acid, $12.5 \mathrm{mg}$; biotin, $0.125 \mathrm{mg}$; folic acid, $1.5 \mathrm{mg}$. ${ }^{2}$ Mineral premix supplied the following as milligram/kilogram of complete feed: zinc, 50; copper, 12; iodine, 0.3 ; cobalt, 0.2 ; iron, 100 ; selenium 0.1 ; manganese, $110 .{ }^{3}$ Concentration of $15 \mathrm{~g} / \mathrm{kg}$ of xanthophylls from Aztec Marigold flower (Tagetes erecta) and $5 \mathrm{~g} / \mathrm{kg}$ of red peppers (Capsicum annum) $10 \mathrm{~g} / \mathrm{kg}$.

Table 2. N-3 and n-6 fatty acid omega $3(n-3)$ and omega 6 (n-6) calculated profile in the soybean and tuna oil used in the experimental diets $(\mathrm{mg} / 100 \mathrm{~g}$ of sample).

\begin{tabular}{ccccccc}
\hline \multirow{2}{*}{ Oil } & \multicolumn{3}{c}{ n-3 Polyunsatured fatty acids } & \multicolumn{3}{c}{ n-6 Polyunsatured fatty acids } \\
\cline { 2 - 7 } & ALA (C18:3) & EPA (C20:5) & DHA (C22:6) & DPA (C22:5) & LA (C18:2) & AA (C20:4) \\
\hline Soybean & 61.95 & 3.94 & 0.24 & 0.15 & 489.70 & 0.02 \\
Tuna & 4.14 & 56.90 & 165.70 & 13.90 & 13.90 & 14.72 \\
\hline
\end{tabular}

ALA = Alpha-linolenic acid, EPA = Eicosapentaenoic acid, DHA = Docosahexaenoic acid, DPA = Docosapentaenoic acid, LA = Linoleic acid, AA Arachidonic acid. 
Table 3. Effect of the substitution of soybean oil with tuna oil on feed intake, egg weight, egg production, and feed conversion of White Leghorn laying hens.

\begin{tabular}{|c|c|c|c|c|c|c|c|c|c|c|c|c|}
\hline \multirow{2}{*}{$\begin{array}{l}\text { Tuna } \\
\text { oil } \%\end{array}$} & \multicolumn{4}{|c|}{ First month } & \multicolumn{4}{|c|}{ Second month } & \multicolumn{4}{|c|}{ Third month } \\
\hline & $\begin{array}{c}\text { Feed Intake } \\
\text { (g/day) }\end{array}$ & $\begin{array}{c}\text { Egg } \\
\text { weight (g) }\end{array}$ & $\begin{array}{c}\text { Laying } \\
(\%)\end{array}$ & $\begin{array}{c}\text { Feed } \\
\text { conversion }\end{array}$ & $\begin{array}{c}\text { Feed Intake } \\
\text { (g/day) }\end{array}$ & $\begin{array}{c}\text { Egg } \\
\text { weight (g) }\end{array}$ & $\begin{array}{c}\text { Laying } \\
(\%)\end{array}$ & $\begin{array}{c}\text { Feed } \\
\text { conversion }\end{array}$ & $\begin{array}{l}\text { Feed Intake } \\
\text { (g/day) }\end{array}$ & $\begin{array}{c}\text { Egg } \\
\text { weight (g) }\end{array}$ & $\begin{array}{l}\text { Laying } \\
(\%)\end{array}$ & $\begin{array}{c}\text { Feed } \\
\text { conversion }\end{array}$ \\
\hline $0 \%$ & $101 \pm 2.0$ & $57 \pm 1.16$ & $92.9 \pm 1.1$ & $1.76 \pm 0.01$ & $109 \pm 2.3$ & $58 \pm 0.55$ & $93 \pm 0.66$ & $1.86 \pm 0.4$ & $114 \pm 0.67$ & $58 \pm 3.89$ & $85 \pm 0.10$ & $2.03 \pm 0.69$ \\
\hline $0.75 \%$ & $105 \pm 1.9$ & $57 \pm 0.81$ & $94.2 \pm 1.1$ & $1.82 \pm 0.02$ & $109 \pm 2.3$ & $59 \pm 0.25$ & $93 \pm 0.26$ & $1.85 \pm 0.03$ & $112 \pm 2.48$ & $55 \pm 0.52$ & $84 \pm 1.82$ & $2.04 \pm 0.04$ \\
\hline $1.50 \%$ & $104 \pm 1.5$ & $57 \pm 0.94$ & $91.8 \pm 1.2$ & $1.84 \pm 0.04$ & $111 \pm 2.5$ & $58 \pm 0.47$ & $92 \pm 1.36$ & $1.91 \pm 0.06$ & $112 \pm 2.43$ & $56 \pm 0.75$ & $86 \pm 0.85$ & $2.03 \pm 0.06$ \\
\hline $2.25 \%$ & $97 \pm 1.8$ & $58 \pm 0.25$ & $93.9 \pm 1.1$ & $1.71 \pm 0.03$ & $108 \pm 1.8$ & $58 \pm 0.73$ & $93 \pm 0.59$ & $1.87 \pm 0.02$ & $109 \pm 1.47$ & $54 \pm 1.11$ & $86 \pm 1.43$ & $2.03 \pm 0.03$ \\
\hline $3 \%$ & $99 \pm 1.6$ & $56 \pm 0.93$ & $91.3 \pm 0.6$ & $1.79 \pm 0.01$ & $108 \pm 0.5$ & $56 \pm 0.60$ & $92 \pm 0.89$ & $1.92 \pm 0.04$ & $120 \pm 1.61$ & $54 \pm 1.08$ & $86 \pm 1.21$ & $2.07 \pm 0.06$ \\
\hline
\end{tabular}

Data is presented as mean \pm standard error. In each column there were no significant differences $(\mathrm{P}>0.05)$.

Table 4. Omega 3 polyunsaturated fatty acids content $(\mathrm{mg} / \mathbf{1 0 0 g})$ of eggs from White Leghorn laying hens fed tuna oil ${ }^{1}$.

\begin{tabular}{|c|c|c|c|c|c|c|}
\hline & Tuna oil $0 \%$ & Tuna oil $0.75 \%$ & Tuna oil $1.50 \%$ & Tuna oil $2.25 \%$ & Tuna oil 3\% & Average \\
\hline \multicolumn{7}{|c|}{$\mathrm{EPA}^{2}(\mathrm{C} 20: 5)$} \\
\hline Month 1 & $3.51 \pm 0.45^{\mathrm{e}}$ & $14.67 \pm 0.85^{\mathrm{d}}$ & $23.88 \pm 1.31^{\mathrm{c}}$ & $36.47 \pm 2.36^{\mathrm{b}}$ & $44.37 \pm 4.16^{\mathrm{a}}$ & $24.57 \pm 2.11$ \\
\hline Month 2 & $3.9 \pm 0.19^{\mathrm{e}}$ & $15.34 \pm 1.95^{\mathrm{d}}$ & $23.32 \pm 1.75^{\mathrm{c}}$ & $34.67 \pm 2.15^{\mathrm{b}}$ & $53.22 \pm 4.66^{\mathrm{a}}$ & $27.57 \pm 2.56$ \\
\hline Month 3 & $3.14 \pm 0.27^{\mathrm{e}}$ & $14.70 \pm 0.99^{\mathrm{d}}$ & $24.07 \pm 1.35^{\mathrm{c}}$ & $33.72 \pm 3.12^{\mathrm{b}}$ & $44.80 \pm 3.49^{\mathrm{a}}$ & $24.57 \pm 2.11$ \\
\hline Average & $3.25 \pm 0.18^{\mathrm{e}}$ & $14.90 \pm 0.76^{\mathrm{d}}$ & $23.79 \pm 0.81^{\mathrm{c}}$ & $34.91 \pm 1.45^{\mathrm{b}}$ & $47.69 \pm 2.44^{\mathrm{a}}$ & \\
\hline \multicolumn{7}{|c|}{ DPA (C22:5) } \\
\hline Month 1 & $74.50 \pm 10.1^{\mathrm{a}}$ & $19.0 \pm 2.9^{b}$ & $8.19 \pm 1.9^{c}$ & $15.45 \pm 2.2^{\mathrm{b}}$ & $20.49 \pm 1.5^{b}$ & $27.21 \pm 3.71$ \\
\hline Month 2 & $47.82 \pm 6.7^{\mathrm{a}}$ & $17.08 \pm 3.8^{\mathrm{b}}$ & $13.17 \pm 2.1^{\mathrm{b}}$ & $15.36 \pm 1.6^{\mathrm{b}}$ & $24.48 \pm 2.1^{\mathrm{b}}$ & $23.68 \pm 2.25$ \\
\hline Month 3 & $60.42 \pm 4.2^{\mathrm{a}}$ & $14.52 \pm 3.1^{\mathrm{b}}$ & $13.75 \pm 1.9^{\mathrm{b}}$ & $15.08 \pm 1.5^{\mathrm{b}}$ & $23.23 \pm 1.5^{\mathrm{b}}$ & $25.20 \pm 2.51$ \\
\hline Average & $60.91 \pm 4.5^{\mathrm{a}}$ & $16.87 \pm 1.9^{\mathrm{b}}$ & $11.52 \pm 1.1^{\mathrm{bc}}$ & $15.30 \pm 0.9^{\mathrm{b}}$ & $22.83 \pm 1.05^{\mathrm{b}}$ & \\
\hline \multicolumn{7}{|c|}{$\mathrm{DHA}^{3}(\mathrm{C} 22: 6)$} \\
\hline Month 1 & $130.86 \pm 6.3^{c}$ & $341.43 \pm 24.0^{\mathrm{b}}$ & $366.82 \pm 31.3^{\mathrm{b}}$ & $501.74 \pm 32.3^{\mathrm{a}}$ & $520.94 \pm 36.7^{\mathrm{a}}$ & $372.27 \pm 21.73$ \\
\hline Month 2 & $113.72 \pm 8.6^{\mathrm{d}}$ & $355.93 \pm 48.3^{\mathrm{c}}$ & $480.56 \pm 38.1^{\mathrm{b}}$ & $452.02 \pm 41.0^{\mathrm{b}}$ & $639.16 \pm 47.4^{\mathrm{a}}$ & $414.81 \pm 28.45$ \\
\hline Month 3 & $128.50 \pm 6.7^{\mathrm{d}}$ & $318.79 \pm 32.8^{c}$ & $425.77 \pm 29.4^{b}$ & $461.20 \pm 40.5^{b}$ & $604.35 \pm 42.3^{\mathrm{a}}$ & $392.40 \pm 24.96$ \\
\hline Average & $124.36 \pm 4.2^{\mathrm{d}}$ & $338.72 \pm 20.6^{c}$ & $419.5 \pm 19.9^{b}$ & $470.38 \pm 22.0^{\mathrm{b}}$ & $591.18 \pm 25.5^{\mathrm{a}}$ & \\
\hline \multicolumn{7}{|c|}{ ALA (C18:3) } \\
\hline Month 1 & $137.95 \pm 8.9^{\mathrm{ab}}$ & $153.11 \pm 9.1^{\mathrm{a}}$ & $110.28 \pm 9.4^{\mathrm{b}}$ & $98.54 \pm 6.26^{\mathrm{bc}}$ & $74.34 \pm 5.1^{\mathrm{cd}}$ & $114.77 \pm 4.99$ \\
\hline Month 2 & $150.40 \pm 6.9^{\mathrm{a}}$ & $127.57 \pm 20.8^{\mathrm{b}}$ & $125.47 \pm 11.7^{\mathrm{b}}$ & $98.65 \pm 6.1^{\mathrm{c}}$ & $83.93 \pm 6.0^{c}$ & $115.26 \pm 5.74$ \\
\hline Month 3 & $139.99 \pm 5.8^{\mathrm{a}}$ & $113.11 \pm 10.1^{\mathrm{b}}$ & $117.36 \pm 7.0^{\mathrm{b}}$ & $86.82 \pm 6.3^{\mathrm{c}}$ & $84.39 \pm 6.5^{c}$ & $107.60 \pm 4.12$ \\
\hline Average & $142.78 \pm 4.2^{\mathrm{a}}$ & $131.26 \pm 8.5^{\mathrm{ab}}$ & $117.04 \pm 5.3^{\mathrm{b}}$ & $94.67 \pm 3.6^{c}$ & $81.13 \pm 3.4^{\mathrm{c}}$ & \\
\hline
\end{tabular}

${ }^{1}$ Lipid content was analyzed in whole eggs (yolk and albumen) as pools of 3 per sample. Data presented as mean \pm standard error. Means in each fatty acid per month with no common superscripts are different $(\mathrm{P}<0.05) .{ }^{2}$ There was a high correlation between the tuna oil inclusion level in the diets and the incorporation of EPA $(\mathrm{r}=0.96, \mathrm{P}<0.05)$ into the egg yolk. ${ }^{3}$ There was a high correlation between the tuna oil inclusion level in the diets and the incorporation of DHA $(\mathrm{r}=$ $0.92, \mathrm{P}<0.05 \%)$ into the egg yolk. 
Table 5. Omega 6 polyunsaturated fatty acids content $(\mathrm{mg} / \mathbf{1 0 0 g})$ of eggs from hens fed tuna oil ${ }^{1}$.

\begin{tabular}{|c|c|c|c|c|c|c|}
\hline & Tuna oil $0 \%$ & Tuna oil $0.75 \%$ & Tuna oil $1.50 \%$ & Tuna oil $2.25 \%$ & Tuna oil 3\% & Average \\
\hline \multicolumn{7}{|l|}{$\mathrm{LA}^{2}(\mathrm{C} 18: 2)$} \\
\hline Month 1 & $1837.38 \pm 123.5^{\mathrm{ab}}$ & $2099.13 \pm 138.4^{\mathrm{a}}$ & $1560.60 \pm 106.8^{b}$ & $1346.3 \pm 70.4^{\mathrm{cd}}$ & $1030.4 \pm 57.7^{\mathrm{d}}$ & $1574.61 \pm 65.55$ \\
\hline Month 2 & $1891.48 \pm 88.0^{\mathrm{a}}$ & $1859.99 \pm 292.6^{\mathrm{a}}$ & $1853.99 \pm 133.6^{\mathrm{b}}$ & $1391.1 \pm 74.1^{\mathrm{b}}$ & $1180.7 \pm 67.9^{b}$ & $1605.83 \pm 75.08$ \\
\hline Month 3 & $1851.73 \pm 85.8^{\mathrm{a}}$ & $1567.73 \pm 132.0^{\mathrm{a}}$ & $1480.83 \pm 90.9^{b}$ & $1215.4 \pm 91.9^{c}$ & $1200.5 \pm 78.4^{\mathrm{c}}$ & $1455.01 \pm 52.14$ \\
\hline Average & $1860.20 \pm 56.5^{a}$ & $1842.28 \pm 118.9^{\mathrm{a}}$ & $1617.07 \pm 66.7^{\mathrm{b}}$ & $1318.8 \pm 46^{\mathrm{bc}}$ & $1141.1 \pm 40.7^{\mathrm{c}}$ & \\
\hline \multicolumn{7}{|l|}{$\mathrm{AA}^{3}(\mathrm{C} 20: 4)$} \\
\hline Month 1 & $304.03 \pm 22.9^{\mathrm{a}}$ & $244.52 \pm 18.2^{\mathrm{a}}$ & $154.59 \pm 7.3^{b}$ & $131.89 \pm 5.7^{b}$ & $106.42 \pm 9.2^{\mathrm{b}}$ & $187.74 \pm 11.32$ \\
\hline Month 2 & $288.10 \pm 16.9^{\mathrm{a}}$ & $224.46 \pm 34.8^{\mathrm{a}}$ & $199.33 \pm 9.48^{b}$ & $134.51 \pm 6.8^{\mathrm{c}}$ & $122.81 \pm 8.2^{\mathrm{c}}$ & $189.46 \pm 11.07$ \\
\hline Month 3 & $300.29 \pm 15.6^{\mathrm{a}}$ & $204.95 \pm 18.0^{\mathrm{b}}$ & $159.81 \pm 9.9^{b}$ & $114.13 \pm 9.4^{\mathrm{c}}$ & $118.71 \pm 8.1^{\mathrm{c}}$ & $177.54 \pm 10.33$ \\
\hline Average & $297.47 \pm 10.5^{\mathrm{a}}$ & $224.64 \pm 14.3^{b}$ & $169.16 \pm 5.9^{c}$ & $126.91 \pm 28.1^{\mathrm{d}}$ & $116.40 \pm 4.91^{\mathrm{d}}$ & \\
\hline
\end{tabular}

${ }^{1}$ Lipid content was analyzed in whole eggs (yolk and albumen) as pools of 3 per sample. Data presented as mean \pm standard error. Means in each fatty acid per month with no common superscripts are different $(\mathrm{P}<0.05) .{ }^{2}$ There was a high correlation between the tuna oil inclusion level in the diets and the incorporation of LA $(\mathrm{r}=-0.95, \mathrm{P}<0.05)$ into the egg yolk. ${ }^{3}$ There was a high correlation between the tuna oil inclusion level in the diets and the incorporation of AA $(\mathrm{r}=$ $-0.96, \mathrm{P}<0.05)$ into the egg yolk.

Table 6. Total n-3 and n-6 polyunsaturated fatty acids content (mg/100g) of eggs from White Leghorn laying hens fed tuna oil ${ }^{1}$.

\begin{tabular}{ccccccc}
\hline & Tuna oil $0 \%$ & Tuna oil $0.75 \%$ & Tuna oil 1.50\% & Tuna oil 2.25\% & Tuna oil 3\% ${ }^{2}$ & Average \\
\hline n-6 PUFA & & & & & & \\
Month 1 & $2141.4 \pm 144.9^{\mathrm{a}}$ & $2343.65 \pm 156.4^{\mathrm{b}}$ & $1715.1 \pm 112.8^{\mathrm{b}}$ & $1478.5 \pm 74.9^{\mathrm{b}}$ & $1136.9 \pm 66.2^{\mathrm{c}}$ & $1762.36 \pm 75.35$ \\
Month 2 & $2179.58 \pm 104.9^{\mathrm{a}}$ & $2084.45 \pm 327.4^{\mathrm{b}}$ & $2053.3 \pm 140.8^{\mathrm{a}}$ & $1525.5 \pm 80.5^{\mathrm{b}}$ & $1303.5 \pm 75.8^{\mathrm{c}}$ & $1795.30 \pm 85.17$ \\
Month 3 & $2152.02 \pm 100.9^{\mathrm{a}}$ & $1772.68 \pm 149.7^{\mathrm{ab}}$ & $1640.65 \pm 99.3^{\mathrm{b}}$ & $1329.5 \pm 100.8^{\mathrm{c}}$ & $1319.2 \pm 86.2^{\mathrm{c}}$ & $1632.56 \pm 61.48$ \\
Average & $2157.67 \pm 66.4^{\mathrm{a}}$ & $2066.93 \pm 133.0^{\mathrm{b}}$ & $1786.24 \pm 71.6^{\mathrm{b}}$ & $1445.75 \pm 50.5^{\mathrm{c}}$ & $1257.5 \pm 45.2^{\mathrm{c}}$ & \\
n-3 PUFA & & & & & & \\
Month 1 & $346.8 \pm 19.1^{\mathrm{c}}$ & $528.2 \pm 36.1^{\mathrm{b}}$ & $524.56 \pm 39.4^{\mathrm{b}}$ & $652.2 \pm 39.4^{\mathrm{a}}$ & $660.16 \pm 47.0^{\mathrm{a}}$ & $542.11 \pm 21.78$ \\
Month 2 & $314.82 \pm 13.3^{\mathrm{d}}$ & $515.94 \pm 73.69^{\mathrm{c}}$ & $642.54 \pm 47.56^{\mathrm{b}}$ & $600.71 \pm 45.8^{\mathrm{b}}$ & $800.8 \pm 59.5^{\mathrm{a}}$ & $580.9 \pm 30.81$ \\
Month 3 & $332.07 \pm 11.7^{\mathrm{d}}$ & $461.14 \pm 46.1^{\mathrm{c}}$ & $580.95 \pm 37.5^{\mathrm{b}}$ & $596.83 \pm 49.0^{\mathrm{b}}$ & $756.79 \pm 53.0^{\mathrm{a}}$ & $549.79 \pm 26.0$ \\
Average & $331.24 \pm 8.72^{\mathrm{d}}$ & $501.77 \pm 30.87^{\mathrm{c}}$ & $577.60 \pm 24.4^{\mathrm{b}}$ & $615.27 \pm 25.8^{\mathrm{b}}$ & $742.80 \pm 31.8^{\mathrm{a}}$ & \\
\hline
\end{tabular}

${ }^{1}$ Lipid content was analyzed in whole eggs (yolk and albumen) as pools of 3 per sample. Data presented as mean \pm standard error. Means in each fatty acid per month with no common superscripts are different $(\mathrm{P}<0.05) .{ }^{2}$ The highest incorporation of total $\mathrm{n}-3 \mathrm{FA}$ content in eggs was obtained with $3 \%$ tuna oil $(\mathrm{r}=0.95$, $\mathrm{P}<0.05)$.

diets up to a concentration of $3 \%$ did not have any detrimental effects on feed consumption, on egg production rate, on egg weight, and on egg feed conversion while producing n-3 fatty acid enriched eggs.

\section{REFERENCES}

[1] W. E. Connor and S. L. Connor, "The Importance of Fish and Docosahexaenoic Acid in Alzheimer Disease," The American Journal of Clinical Nutrition, Vol. 85, No. 4,
2007, pp. 929-930.

[2] A. P. Simopoulos, "The Omega-6/Omega-3 Fatty Acid Ratio, Genetic Variation, and Cardiovascular Disease," Asia Pacific Journal of Clinical Nutrition, Vol. 17, Suppl. 1, 2008, pp. 131-134.

[3] A. P. Simopoulos, "Evolutionary Aspects of the Dietary Omega-6:Omega-3 Fatty Acid Ratio: Medical Implications," World Review of Nutrition and Dietetics, Vol. 100, 2009, pp. 1-21. doi:10.1159/000235706

[4] A. P. Simopoulos, "Omega-6/Omega-3 Essential Fatty 
Acids: Biological Effects," World Review of Nutrition and Dietetics, Vol. 100, 2009, pp. 1-16. doi: $10.1159 / 000235706$

[5] C. Nishida and R. Uauy, "WHO Scientific Update on Health Consequences of Trans Fatty Acids: Introduction," European Journal of Clinical Nutrition, Vol. 63, Suppl. 2, 2009, pp. S1-S4. doi:10.1038/ejen.2009.13

[6] P. S. Hargis, M. E. Van Elswyk and B. M. Hargis, "Dietary Modification of Yolk Lipid with Menhaden Oil," Poultry Science, Vol. 70, No. 4, 1991, pp. 874-883. doi: $10.3382 /$ ps. 0700874

[7] A. C. Marshall, K. S. Kubena, K. R. Hinton, P. S. Hargis and M. E. Van Elswyk, "N-3 Fatty Acid Enriched Table Eggs: A Survey of Consumer Acceptability," Poultry Science, Vol. 73, No. 8, 1994, pp. 1334-1340. doi:10.3382/ps.0731334

[8] M. D. Baucells, N. Crespo, A. C. Barroeta, S. LopezFerrer and M. A. Grashorn, "Incorporation of Different Polyunsaturated Fatty Acids into Eggs," Poultry Science, Vol. 79, No.1, 2000, pp. 51-59.

[9] P. R. Howe, J. A. Downing, B. F. Grenyer, E. M. Grigonis-Deane and W. L. Bryden, "Tuna Fishmeal as a Source of DHA for n-3 PUFA Enrichment of Pork, Chicken, and Eggs," Lipids, Vol. 37, No. 11, 2002, pp. 1067-1076. doi:10.1007/s11745-002-1002-3

[10] J. M. Bourre, "Effect of Increasing the Omega-3 Fatty Acid in the Diets of Animals on the Animal Products Consumed by Humans," Medécine Sciences (Paris), Vol. 21, No. 8-9, 2005, pp. 773-779. doi:10.1051/medsci/2005218-9773

[11] S. Carrillo-Dominguez, M. E. Carranco-Jauregui, R. M. Castillo-Dominguez, M. I. Castro-Gonzalez, E. AvilaGonzalez and F. Perez-Gil, "Cholesterol and n-3 and n-6 Fatty Acid Content in Eggs from Laying Hens Fed with Red Crab Meal (Pleuroncodes planipes)," Poultry Science, Vol. 84, No. 1, 2005, pp. 167-172.

[12] National Research Council, "Nutrient Requirements of
Poultry," 9th revised Edition, National Academy Press, Washington DC, 1994.

[13] J. Folch, M. Lees and G. H. Sloane Stanley, "A Simple Method for the Isolation and Purification of Total Lipides from Animal Tissues," The Journal of Biological Chemistry, Vol. 226, No. 1, 1957, pp. 497-509.

[14] M. I. Castro González, S. Montano Benavides and F. Pérez-Gil Romo, "Fatty Acids in Sardine Canned in Tomato Sauce from Different Fishing Areas of the Mexican Pacific," Archivos Latinoamericanos de Nutrición, Vol. 51, No. 4, 2001, pp. 400-406.

[15] SAS Institute Inc, "SAS User's Guide: Statistics," SAS Institute Inc., Cary, 2002.

[16] A. P. Simopoulos, "Commentary. Genetic Variants and Omega-6, Omega-3 Fatty Acids: Their Role in the Determination of Nutritional Requirements and Chronic Disease Risk," Journal of Nutrigenetics and Nutrigenomics, Vol. 2, No. 3, 2009, pp. 117-118. doi: $10.1159 / 000235630$

[17] P. Bovet, D. Faeh, G. Madeleine, B. Viswanathan and F. Paccaud, "Decrease in Blood Triglycerides Associated with the Consumption of Eggs of Hens Fed with Food Supplemented with Fish Oil," Nutrition, Metabolism and Cardiovascular Diseases, Vol. 17, No. 4, 2007, pp. 280287. doi:10.1016/j.numecd.2005.12.010

[18] C. Vanek and W. E. Connor, "Do n-3 Fatty Acids Prevent Osteoporosis?" The American Journal of Clinical Nutrition, Vol. 85, No. 3, 2007, pp. 647-648.

[19] B. Burlingame, C. Nishida, R. Uauy and R. Weisell, "Fats and Fatty Acids in Human Nutrition: Introduction," $\mathrm{An}$ nals of Nutrition Metabolism, Vol. 55, No. 1-3, 2009, pp. 5-7. doi:10.1159/000228993

[20] R. Uauy and A. D. Dangour, "Fat and Fatty Acid Requirements and Recommendations for Infants of $0-2$ Years and Children of 2 - 18 Years," Annals of Nutrition and Metabolism, Vol. 55, 2009, pp. 76-96. doi:10.1159/000228997 